\title{
Latency and Capacity Optimal Broadcasting in Wireless Multihop Networks with Arbitrary Number of Sources
}

\author{
Giovanni Resta, and Paolo Santi
}

\begin{abstract}
This paper studies the fundamental properties of broadcasting in multi-hop wireless networks. Previous studies have shown that, as long as broadcast capacity is concerned, asymptotically optimal broadcasting is possible in wireless multihop networks under very general conditions. However, none of the existing work on broadcast capacity has considered latency in message delivery, which is simply assumed to be finite (but not explicitly bounded). In this paper, the issue of investigating the fundamental properties of broadcast communications for what concerns both capacity and latency using a realistic, SINRbased interference model is investigated. In particular, a novel topological notion of network connectivity is introduced, and it is shown that, if the network satisfies this property, asymptotically optimal broadcast capacity and latency can be achieved simultaneously. The above result holds in the general scenario in which an arbitrary number of broadcast sources arbitrarily share the available (optimal) network capacity. The result presented in this paper is in sharp contrast to similar results obtained for the case of unicast transmissions, where asymptotically optimal latency in message delivery can be achieved only at the expense of asymptotically reducing network capacity. Thus, the results presented in this paper show that scalable broadcasting in multihop wireless networks is, in principle, possible.
\end{abstract}

Index Terms-broadcast capacity; broadcast latency; cell connectivity; SINR interference model; wireless networks.

\section{INTRODUCTION}

Investigation of fundamental properties of wireless multihop networks has received considerable attention in the research community, since it can help understanding what can and cannot be done in such networks. In their seminal work [10], Gupta and Kumar investigate the asymptotic network capacity of a wireless multi-hop network for unicast transmissions, and show that network capacity does not scale: as the number $n$ of network nodes increases, the per-node available capacity decreases as $O(1 / \sqrt{n})$ in arbitrary networks, and as $O(1 / \sqrt{n \log n})$ in random networks ${ }^{1}$. This lack of scaling of network capacity is due to the relay burden on intermediate nodes caused by the multi-hop nature of communications: due to interference, the node transmission ranges must be kept

G. Resta and P. Santi are with the Istituto di Informatica e Telematica del CNR, Pisa, Italy.

A preliminary version of this paper appeared on the Proceedings of the IEEE Int. Conference on Communications (ICC), 2009.

${ }^{1}$ In this paper, we will extensively use the big-O and similar notation for describing asymptotic behavior of functions. Formally, given two functions $f(x)$ and $g(x)$, we have: $f(x)=O(g(x))$ if and only if $\lim _{x \rightarrow \infty} \frac{f(x)}{g(x)}<$ $\infty ; f(x)=\Omega(g(x))$ if and only if $\lim _{x \rightarrow \infty} \frac{g(x)}{f(x)}<\infty$; and $f(x)=$ $\Theta(g(x))$ if and only if $f(x)=O(g(x))$ and $f(x)=\Omega(g(x))$. limited; hence, a packet must travel several hops in order to reach the destination. Thus, a single transmission results in a series of relay re-transmissions interfering both with other packets on the same flow, and with packets on different flows. For this reason, we say that unicast transmissions in a wireless multi-hop network are relay limited. Note that use of relay nodes not only limits capacity, but it also has a negative impact on latency, which is increased with respect to the case of direct, single-hop transmission.

In [9], Grossglauser and Tse proved that mobility can be used to lessen the relay burden and achieve optimal network capacity scaling (i.e., $\Theta(W)$ per-node capacity, where $W$ is the channel capacity). However, the capacity achieving scheduling and routing schemes defined in [9] assume unlimited buffer capacity on the nodes. Furthermore, Grossglauser and Tse results do not consider latency in message delivery, which can grow unboundedly. More recently, Ozgur et al. [16] argued that $\Omega(W)$ per-node capacity can indeed be achieved also in a static network. The idea is to use hierarchical cooperation among network nodes aimed at realizing a distributed MIMO communication. However, this result is quite controversial, since it implicitly assumes availability of an arbitrarily large number of independent information channels between group of nodes. Franceschetti et al. [7] have shown that the actual number of independent channels across two regions in a twodimensional domain is indeed upper bounded by $O(\sqrt{n})$, which implies a $O\left((\log n)^{2} / \sqrt{n}\right)$ per-node capacity in case of random networks. Note that, similar to [9], latency in packet delivery is not considered in [7], [16].

Since these seminal works, many authors have tried to gain a better understanding of the fundamental tradeoffs between network capacity and latency in packet delivery [1], [15], [23], [24]. The tradeoff stems from the fact that, in order to reduce latency, relatively long transmission ranges should be used, with optimal latency achieved with direct, singlehop communications. On the other hand, as the results of [9], [10] have shown, using long transmission ranges is suboptimal for what concerns capacity, which is optimized when nodes use very short transmission ranges. In [4], [5], the authors show that the optimal tradeoff for static networks is achieved when $D(n)=\Theta(n T(n))$, where $T(n)$ is the average per-node throughput in a network with $n$ nodes, and $D(n)$ is the average packet delay ${ }^{2}$. Note that this result

\footnotetext{
${ }^{2}$ In this paper, terms per-node throughput and per-node capacity, as well as terms latency and delay, are used interchangeably.
} 
implies that $\Omega(W)$ (i.e., optimal) per-node throughput can be achieved only by allowing relatively high $(O(n))$ packet delay. The same throughput/delay tradeoff can be achieved also with hierarchical cooperation [17]. In case of mobile networks, in [4] it is shown that the optimal tradeoff remains $D(n)=\Theta(n T(n))$ as long as $T(n)=O(1 / \sqrt{n \log n})$, i.e., when the per-node throughput does not exceed the one achieved in static networks. If higher throughput values are sought, the achievable delay becomes $\Theta(n \log n)$, and worse throughput/delay tradeoff is achieved. The worse tradeoff in case of mobile networks can be explained observing that higher throughput than in static networks can be obtained only by exploiting physical node mobility which, however, is several orders of magnitude slower than the speed of radio propagation in the air. Thus, exploiting physical node mobility to increase throughput causes a significant degradation of delay performance.

Investigation of fundamental properties of broadcast communications has received attention from the research community only very recently. In [25], Zheng investigated the broadcast capacity for the case of random networks with single broadcast source under the generalized physical interference model, and presented a broadcast scheme providing a capacity within a constant factor from optimal. The authors of [13] considered a more general network model, in which arbitrary node positions are allowed, and an arbitrary subset of the network nodes is assumed to generate broadcast packets. The results of [13] confirms the findings of [25], i.e., that the (aggregate) broadcast capacity is within a constant factor from the optimal capacity. More recently, the same authors have proved in [12] that the same result holds using more realistic interference models, namely the physical and the generalized physical interference model. However, none of the existing work on broadcast capacity has investigated the latency in message delivery. More specifically, in the network models used in [12], [13], [25] it is assumed that broadcast packets are eventually received by all network nodes, but no explicit nor implicit upper bound on delivery time is given. Zheng in [25] also investigated the information diffusion rate, which is closely related to latency, and provides matching upper and lower bounds for this quantity. However, the broadcast schemes used for lower bounding broadcast capacity and information diffusion rate are different, and the problem of finding an optimal broadcasting scheme for both capacity and information diffusion rate in the model of [25] remains open.

The above discussion highlights that the fundamental question of whether asymptotically optimal capacity and latency can be achieved simultaneously in case of broadcast communications remains, to the best of our knowledge, open.

In this paper, we give a positive answer to this question by showing that, at least under certain, quite general, assumptions on node deployment, asymptotically optimal capacity and latency can indeed be achieved simultaneously. More specifically, we restrict our attention to a network setting in which optimal per-node capacity can be achieved. This network setting naturally imposes a lower bound $\underline{D}(n)$ on the achievable delay performance, mainly due to the fact that a certain value of the transmission range is used to optimize per-node capacity. The main result of this paper is presenting a broadcasting scheme that simultaneously achieves optimal (i.e, $\Omega(W)$ ) per-node capacity and latency $D(n)=\Theta(\underline{D}(n))$, which is then optimal under the assumption of maximizing capacity.

To derive the above result, we introduce a novel topological notion of network connectivity which we call cell connectivity, and show that asymptotically optimal broadcast capacity and latency can be achieved simultaneously (in the sense explained above) if the network is cell connected. Our result is proved using the realistic physical interference model of [10], and holds when an arbitrary number of broadcast sources arbitrarily share the available broadcast capacity. Thus, in sharp contrast with the case of unicast transmissions, our result suggests that broadcasting is not relay limited, and scalable broadcasting in multi-hop wireless networks is, in principle, possible.

We stress that latency is a fundamental parameter of broadcast communications, at least in some scenarios such as multimedia and real-time applications. For instance, if a wireless multi-hop network is used for communication of multimedia information among members of a disaster rescue team.

The problem of latency optimal broadcasting (with no consideration on broadcast capacity, though) is studied, for instance, in [8], and, more recently, in [11], where the authors study the problem also under the physical interference model.

\section{NETWORK MODEL AND PRELIMINARIES}

We consider a wireless network composed of $n$ wireless nodes distributed in a two-dimensional domain. We assume nodes communicate through a shared wireless channel of a certain, constant capacity $W$, and that the nodes transmission power is fixed to some value $P$. Correct message reception at a receiver node is subject to a SINR-based criterion, also known as physical interference model [10]. More specifically, a packet sent by node $u$ is correctly received at a node $v$ (with rate $W$ ) if and only if

$$
\frac{P_{v}(u)}{N+\sum_{i \in \mathcal{T}} P_{v}(i)} \geq \beta,
$$

where $N$ is the background noise, $\beta$ is the capture threshold, $\mathcal{T}$ is the set of nodes transmitting concurrently with node $u$, and $P_{v}(x)$ is the received power at node $v$ of the signal transmitted by node $x$.

We also make the standard assumption that radio signal propagation obeys the log-distance path loss model [20], according to which the received signal strength at distance $d$ from the transmitter (for sufficiently large $d$, say, $d \geq 1$ ) equals $P \cdot d^{-\alpha}$, where $\alpha$ is the path loss exponent. In the following, we make the standard assumption that $\alpha>2$, which is often the case in practice. We then have ${ }^{3} P_{v}(x)=P \cdot d(x, v)^{-\alpha}$, where $d(x, v)$ is the Euclidean distance between nodes $v$ and $x$, and the SINR value at node $v$ can be rewritten as follows

$$
\operatorname{SINR}(v)=\frac{d(u, v)^{-\alpha}}{\frac{N}{P}+\sum_{i \in \mathcal{T}} d(i, v)^{-\alpha}} .
$$

\footnotetext{
${ }^{3}$ To simplify notation, in the following we assume that the product of the transmitter and receiver antenna gain is 1 .
} 
For given values of $P, \beta, \alpha$, and $N$, we define the transmission range $r_{\max }$ of a node as the maximum distance up to which a receiver can successfully receive a message in absence of interference. From the definition of physical interference model, we have:

$$
r_{\max }=\sqrt[\alpha]{P /(\beta N)} .
$$

The maximal communication graph is a graph $G_{M}=$ $\left(\mathcal{V}, \mathcal{E}_{\mathcal{M}}\right)$ representing all possible communication links in the network, i.e., $\mathcal{V}$ is the set of the $n$ nodes, and (undirected) edge $(u, v) \in \mathcal{E}$ if and only if $d(u, v) \leq r_{\max }$.

Given an arbitrary set $\mathcal{S} \subseteq \mathcal{V}$ of broadcast source nodes in a network with $n$ nodes, and denoting with $\lambda(s)$ the rate at which source $s \in \mathcal{S}$ generates packets, we define the broadcast capacity of the network as the maximum possible feasible aggregate rate $\lambda(n)=\sum_{s \in \mathcal{S}} \lambda(s)$. Rate $\lambda(n)$ is feasible if and only if all packets generated by source $s \in \mathcal{S}$ are received by all nodes in $\mathcal{V}-\{s\}$ within a certain time $T_{\max }$, with $T_{\max }<$ $\infty$. Observe that the aggregate capacity $\lambda(n)$ is expressed as a function of the number $n$ of nodes, and not of the set $\mathcal{S}$ of broadcast sources. This is because all the results presented in this paper, once fixed the number $n$ of network nodes, hold for arbitrary broadcast source sets. To simplify presentation, in the first part of the paper we focus our attention on the case of single broadcast source, i.e., $\mathcal{S}=\{s\}$, for some $s \in \mathcal{V}$. Then, we show how our results can be extended to the case of arbitrary broadcast source sets.

Our results indeed hold under a more general model, which includes proportional fairness in the definition of broadcast capacity. More specifically, a bandwidth allocation vector $\mathcal{B}=$ $\left(b_{1}, \ldots, b_{n}\right)$ representing the proportional bandwidth allocated to each broadcast source (where $b_{i}=0$ means that node $i$ is not a broadcast source), with $\sum_{i=1}^{n} b_{i}=1$, is given in input. The proportionally fair broadcast capacity of the network is defined by the maximum weight $\delta(n)$ such that the aggregate broadcast rate $\lambda(\mathcal{B}, \delta(n))=\sum_{i=1}^{n} b_{i} \cdot \delta(n)$ is feasible.

The broadcast latency of the network is the minimal time $T(n)$ such that, for all possible sources $s \in \mathcal{S}$, all nodes in $\mathcal{V}-\{s\}$ receives a packet generated by $s$ at time $t$ within time $t+T(n)$. It is clear that, in order to have meaningful values of broadcast capacity and latency, the maximal communication graph of a network must be connected. Thus, the assumption of connected maximal communication graph is made throughout this paper.

\section{Cell CONNECTED NeTWORKS}

In order to obtain non-trivial bounds for the broadcast latency, some assumptions on network deployment must be made. In fact, while broadcast capacity is somewhat independent of the shape of the deployment region and node positions as long as the maximal communication graph is connected (see, e.g., [12]), broadcast latency depends on the distance between the broadcast source and the farthest node, which, in turn, depends on how nodes are deployed in the plane.

In the following we introduce the notion of cell connectivity, which is sufficient to obtain asymptotically optimal upper bounds to broadcast latency. Our results are valid for any
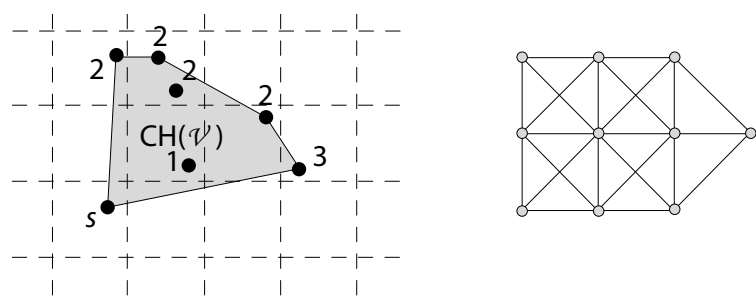

Fig. 1. Example of network deployment (left), and corresponding cell graph (right). The convex hull of the network nodes is shaded. Network nodes on the left are labeled with their cell distance to node $s$.

network deployment (i.e., maximal communication graph) satisfying cell connectivity. In particular, in this section we show that a widely investigated class of network deployments, known as random networks or homogeneous networks and used, e.g., in [4], [5], [7], [10], [16], [17], satisfies the conditions for cell connectivity w.h.p. ${ }^{4}$. Thus, the results presented in this paper are valid for a quite general class of network deployments including random network deployments.

Before introducing cell connectivity, we define a square lattice of the plane, and introduce the notion of cell distance between an arbitrary pair of network nodes.

Definition 1 (Cell graph): Assume the plane is partitioned into a lattice of square cells of side $l$, with $l=\frac{r_{\max }}{2 h \sqrt{2}}$, for some constant $h>1$, and let $v_{1}, \ldots, v_{n}$ denote the positions of the $n$ network nodes in the plane. The cell graph of the network is the graph $C G=(\mathcal{C}, \mathcal{E C})$, with a vertex corresponding to each cell $c$ such that $c \cap C H(\mathcal{V}) \neq \emptyset$, with $C H(\mathcal{V})$ denoting the convex hull of points $v_{1}, \ldots, v_{n}$, and undirected edge $(x, y) \in$ $\mathcal{E C}$ if and only if cells $x, y$ are adjacent (horizontal, vertical, and diagonal adjacency).

Definition 2 (Cell distance): Let cell $(x)$ denotes the cell to which node $x \in \mathcal{V}$ belongs to. The cell distance $c d(u, v)$ between nodes $u, v \in \mathcal{V}$ is defined as the hop distance between the corresponding vertexes $\operatorname{cell}(u)$ and $\operatorname{cell}(v)$ in the cell graph.

An example of network deployment and correspondent cell graph and distances is shown in Figure 1. Note that the condition on $l$ relative to $r_{\max }$ is needed to ensure the following claim holds:

Claim 1: Let $u$ be an arbitrary node located in cell cell $(u)$; all the nodes located in the 8 cells adjacent to $\operatorname{cell}(u)$ (horizontal, vertical, and diagonal adjacency) are within distance $r_{\max }$ from $u$.

In the following, we use the term communication graph (or, simply, graph) to refer to an arbitrary subgraph $G=(\mathcal{V}, \mathcal{E})$ of the maximal communication graph $G_{M}$. Intuitively speaking, a communication graph represents the set of links used by a certain communication scheme, which does not necessarily coincide with the set of all possible links in the network. Unless otherwise stated, in the following $(u, v) \in \mathcal{E}$ denotes a directed transmission link between node $u$ (the sender, or transmitter, node) and node $v$ (the receiver node).

\footnotetext{
${ }^{4}$ In this paper, w.h.p. means with probability approaching 1 as $n \rightarrow \infty$. While stronger notions of w.h.p. are sometimes used in the literature (e.g., requiring the probability to converge to 1 as $1-O(1 / n))$, the relatively weak notion of w.h.p. used in this paper is sufficient for our purposes.
} 

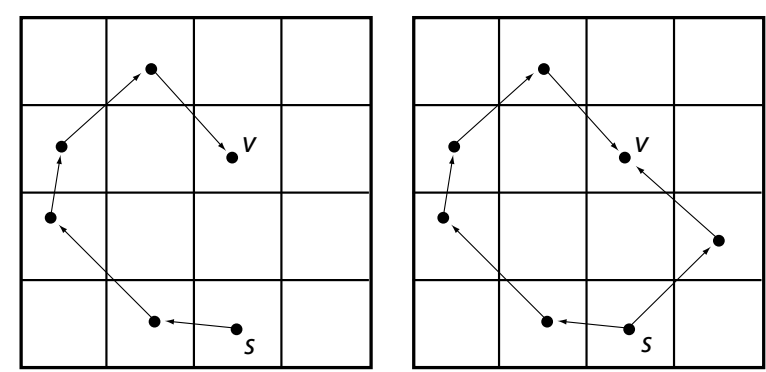

Fig. 2. The notion of cell connectivity.

Definition 3 (Cell connectivity): Let $G=(\mathcal{V}, \mathcal{E})$ be an arbitrary communication graph. Graph $G$ is cell connected with respect to node $s \in \mathcal{V}$ if and only if, for any node $v \in \mathcal{V}-\{s\}$, there exists at least one path $P$ connecting $s$ and $v$ in $G$ such that for all nodes $w$ in $P-\{v\}$, we have $c d(s, w)<c d(s, v)$.

The notion of cell connectivity is pictorially explained in Figure 2: the network on the left is not cell connected w.r.t. $s^{5}$, since the only path connecting $s$ and $v$ contains nodes whose cell distance to $s$ is greater than $\operatorname{cd}(s, v)-1=1$. On the contrary, the network on the right is cell connected, since the rightward path connects $s$ to $v$ through a node whose cell distance to $s$ is less than $c d(s, v)$. As we shall see, the notion of cell connectivity is fundamental to ensure an asymptotically optimal progress of broadcast packets generated at $s$ towards nodes in $\mathcal{V}-\{s\}$, i.e., optimal broadcast latency. Note also that the notion of cell connectivity is a monotonic graph property, i.e., $G^{\prime} \subseteq G$ and $G^{\prime}$ is cell connected implies that also graph $G$ is cell connected, where $G$ is any super-graph of $G^{\prime}$ obtained by adding edges (but not nodes) to $G^{\prime}$.

Definition 4 (Strong cell connectivity): Let $G=(\mathcal{V}, \mathcal{E})$ be an arbitrary communication graph. Graph $G$ is strongly cell connected if and only if it is cell connected with respect to any node $s \in \mathcal{V}$.

Definition 5 (Cell adjacency): A communication graph $G=(\mathcal{V}, \mathcal{E})$ is said to satisfy the cell adjacency property if and only if set $\mathcal{E}$ is a superset of the set of links $(u, v)$ with nodes $u, v$ belonging to either adjacent (horizontal, vertical, and diagonal adjacency) cells or to the same cell.

In other words, the cell adjacency property implies that all links whose endpoints lie in the same or adjacent cells are part of the communication graph.

Given the above property, the following claims are trivial to show:

Claim 2: If every cell in the cell graph corresponding to a certain communication graph $G=(\mathcal{V}, \mathcal{E})$ satisfying cell adjacency contains at least one node, then graph $G$ is strongly cell connected.

Claim 3: If every empty cell in the cell graph corresponding to a certain communication graph $G=(\mathcal{V}, \mathcal{E})$ satisfying cell adjacency is adjacent only to non-empty cells, then graph $G$ is strongly cell connected.

Note that the number and patterns of empty cells depends on the step $l$ of the square lattice used to partition the plane.

\footnotetext{
${ }^{5}$ When the actual node $s$ w.r.t. a graph $G$ is cell connected is not relevant, we will simply say that $G$ is "cell connected".
}

Intuitively speaking, fixed arbitrary positions of the network nodes, the larger the step $l$ of the square lattice, the less likely it is to have empty cells. On the other hand, the larger the value of $l$, the smaller the cell distance between arbitrary nodes tends to be, hindering existence of "cell connected" paths in the communication graph. We have verified through simulations with random uniform node deployment that, even for low density scenarios - i.e., regimes in which the transmission range is below the critical value for connectivity w.h.p. the vast majority (above 98\%) of networks such that the maxpower communication graph is connected are also strongly cell connected.

To ease presentation, in the following we consider a network to be cell connected w.r.t. a certain node $s$ if there exists at least one square lattice partitioning of side $\bar{l}=\frac{r_{\max }}{2 \bar{h} \sqrt{2}}$, where $\bar{h}$ is a constant greater than 1 , such that the network is cell connected w.r.t. node $s$ for that specific lattice. This specific square lattice (or one of them, in case the network is cell connected for more than one square lattices) is assumed to be used in the derivation of optimal broadcast capacity and latency bounds.

We conclude this section with the proof that a relevant network deployment scenario, known as homogeneous networks or random networks in the literature, satisfies strong cell connectivity. More specifically, we consider the following node deployment scenario:

a1. a number $n$ of nodes is distributed uniformly at random in a square region ${ }^{6}$ of side $\left(r_{\max } / j\right) \cdot \sqrt{n / \log n}$, where $j>1$ is a constant defined in Lemma 1 .

In the above setting, the critical transmission range for connectivity $\operatorname{ctr}(n)$, i.e., the minimal value of the transmission range such that the resulting network topology is connected w.h.p. (this is a necessary condition for having meaningful notions of broadcast capacity and latency) is [6], [12]:

$$
\operatorname{ctr}(n)=\sqrt{\left(\frac{r_{\max }}{j}\right)^{2} \cdot \frac{n}{\log n} \cdot \frac{\log n}{n}}=\frac{r_{\max }}{j},
$$

i.e., it is a constant fraction of the maximum transmission range. In other words, we are considering a situation in which node density (defined here as the average number of nodes within transmission range) is minimal (up to a constant factor) for obtaining network connectivity w.h.p.

Let us now assume the deployment region is divided into $C=\frac{8 h}{j} \cdot \frac{n}{\log n}$ non-overlapping square cells of side $l=\frac{r_{\max }}{2 h \sqrt{2}}$, where $h>1$ is an arbitrary constant. We now prove a quite standard concentration result showing that, under the above conditions, all the cells contains at least one node w.h.p., which, by Claim 2, implies strong cell connectivity under the assumption that the communication graph satisfies cell adjacency. The interested read can find the formal proof of the lemma in the Appendix.

Lemma 1: Assume $n$ nodes are distributed uniformly at random in a square region of side $\frac{r_{\max }}{j} \cdot \sqrt{\frac{n}{\log n}}$, for some

\footnotetext{
${ }^{6} \mathrm{Up}$ to straightforward technical details, this result can be extended to the case where the nodes are distributed in an arbitrary convex region of volume $\left(\frac{r_{\max }}{j}\right)^{2} \cdot \frac{n}{\log n}$.
} 
arbitrary constant $j>1$, and that the deployment region is divided into $C=\frac{8 h^{2}}{j^{2}} \cdot \frac{n}{\log n}$ non-overlapping square cells of side $l=\frac{r_{\max }}{2 h \sqrt{2}}$. If $j^{2} \geq 8 h^{2}$, then the minimally occupied cell contains at least one node, w.h.p.

Corollary 1: Assume $a 1$; if a communication graph $G$ satisfies cell adjacency, then $G$ is strongly cell connected w.h.p.

\section{TRIVIAL BOUNDS ON BROADCAST CAPACITY AND LATENCY}

The following upper bound on the broadcast capacity trivially follows by observing that the maximum rate at which any receiver can receive broadcast packets is $W$ [12]. The bound holds for an arbitrary network and arbitrary broadcast source sets.

Claim 4: In any network with $n$ nodes, we have $\lambda(n) \leq W$.

Define $d(n)$, the diameter of the network, as the maximum Euclidean distance between any two network nodes $u, v \in \mathcal{V}$. The lower bound on the broadcast latency immediately follows by the definition of transmission range $r_{\max }$. Also this bound holds for an arbitrary network and arbitrary broadcast source sets.

Claim 5: Given any network with $n$ nodes, we have $T(n) \geq \frac{d(n)}{r_{\max }}=\underline{D}(n)$.

\section{MATChing CAPACITY AND LATENCY BOUNDS: THE SINGLE SOURCE CASE}

In this section, we introduce a broadcast scheme for single source broadcasting based on a $k^{2}$ coloring of a subset of network nodes, which enjoys the following properties: $i$ ) the broadcast source $s$ generates new packets at rate $\Omega\left(\frac{W}{k^{2}}\right)$; and ii) all generated packets are correctly received by all nodes in $\mathcal{V}-\{s\}$ within time $O\left(\frac{d(n)}{r_{\max }}\right)$ under the condition that a properly defined communication graph $G_{k}$ (see below for a formal definition) is cell connected w.r.t. source node $s$.

Note that, in order to have asymptotically optimal broadcast capacity, the number $k^{2}$ of colors used by the broadcast scheme must be a constant. On the other hand, we need that the communication graph satisfies cell connectivity in order to have asymptotically optimal latency. We shall see that, fixed a step $l$ of the square lattice used to partition the plane, and two possible values $k^{\prime}$ and $k$ of the coloring scheme, $k^{\prime}<k$ implies that communication graph $G_{k^{\prime}}$ used to broadcast with $\left(k^{\prime}\right)^{2}$ colors is a subgraph of the communication graph $G_{k}$ used to broadcast with $k^{2}$ colors. Given that cell connectivity is a monotonic graph property, $G_{k^{\prime}} \subseteq G_{k}$ implies that, from the point of view of cell connectivity (which is a sufficient condition to show asymptotically optimal broadcast latency), a relatively large value of $k$ is desirable. In other words, if we select a relatively low value of $k$, it is relatively more likely that the resulting communication graph $G_{k}$ is not cell connected, and optimal latency in packet delivery cannot be ensured. On the other hand, a very large value of $k$ (say, $k=f(n)$, where $f(n)$ is some unbounded increasing function of $n$ ) is likely to result in a cell connected communication graph, but it is not optimal for what concern broadcast capacity. Thus, the choice of the number of colors $k^{2}$ in the
Algorithm for a generic node $v$ :

Let $i$ be the color of the current time slot

If $v$ is a leader node, let $j$ be the ID of the

last packet transmitted by node $v$

1. if $\operatorname{color}(v)=i$ then

2. if $\operatorname{source}(v)$ then transmit new packet

3. else if cellLeader $(v)$ then

4. if $\operatorname{buffer}(v)$ is not empty then

5. transmit packet and empty buffer

6. else $/ / \operatorname{color}(v) \neq i$

7. if not $\operatorname{source}(v)$ then

8. listen to the channel

9. if new packet arrive then

10. receive the packet

11. let $j^{\prime}$ be the ID of the received packet

12. if $($ cellLeader $(v))$ and $\left(j^{\prime}=j+1\right)$ then

13. store packet in transmit buffer

Fig. 3. The broadcasting scheme with single broadcast source.

coloring scheme must fulfill opposite requirements for what concerns broadcast capacity (which would require minimizing $k$ ) and latency (which would require maximizing $k$ ).

In this section, we show how to address this tradeoff, by showing that there exists a constant value $\bar{k}$ such that communication graph $G_{\bar{k}}$ satisfies cell adjacency, which ensures that cell connectivity of graph $G_{\bar{k}}$ can be guaranteed for the relevant network deployments characterized in claims 2 and 3 and Corollary 1 (e.g., for random networks). We recall that a constant value of the number of color guarantees optimal broadcast capacity. Thus, setting $k=\bar{k}$ simultaneously achieve asymptotically optimal broadcast capacity and latency bounds. More in general, our characterization shows that asymptotically optimal broadcast capacity and latency is achievable whenever communication graph $G_{\bar{k}}$ is cell connected w.r.t. the source node, which can occur also when none of the conditions necessary for claims 2 and 3 and Corollary 1 holds.

To prove our result we use the simple broadcast scheme reported in Figure 3. We assume that the plane is partitioned into non-overlapping square cells of side $l$, that each node $v$ is aware of the cell $\operatorname{cell}(v)$ to which it belongs, and that a spatial TDMA scheme is used at the MAC layer. Each node $v$ in the network is assigned with a color color $(v)$ chosen among a set of $k^{2}$ colors. Details of the coloring scheme, which is similar to the ones used, e.g., in [12], [25], are given below. Time is divided into periods composed of $k^{2}$ transmission slots, one for each color. We assume slot coloring is periodic with period $k^{2}$, i.e., if slot $j$ has color $i$, then also slots $j+z k^{2}$, for any integer $z \geq 1$, have color $i$. All nodes have a single-packet transmit buffer; i.e., when a new packet arrives, if the previous packet has not yet been sent, the old packet is overwritten.

The source node $s$ simply transmits a new packet each time a slot of color color $(s)$ occurs. Leader nodes are selected arbitrarily in each populated cell. If a node $v$ is selected as cell leader, i.e., as the only node in $\operatorname{cell}(v)$ responsible for forwarding packets, then $v$ transmits a packet whenever a slot of color color $(v)$ occurs, subject to the condition that there is a new packet to send in the buffer. Each non-source node listens to the channel in the remaining $k^{2}-1$ slots of the period and, in case a new packet is received and the node is a cell leader, the new packet is stored in the buffer (possibly overwriting an 


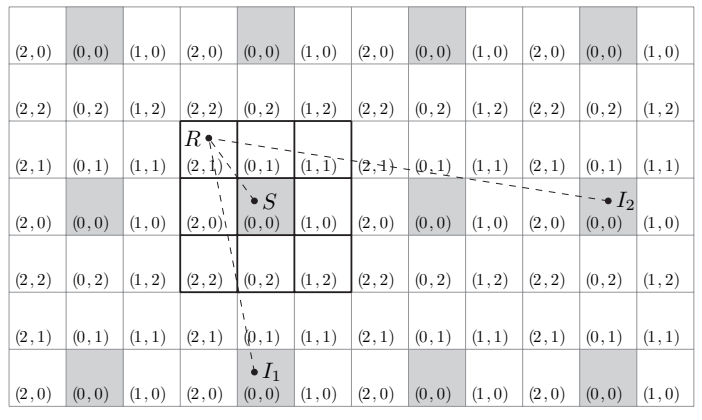

Fig. 4. Two-dimensional coloring of parameter $k=3$.

old packet). Note that new packets can be easily identified by a sequential packet ID contained in the header of the packet. Condition $j^{\prime}=j+1$ at step 12. ensures that the broadcasting scheme preserves packet ordering, i.e., single packet buffering at the leader nodes is sufficient (see Theorem 1 for details).

The coloring scheme is as follows. We recall that we assume a square lattice partitioning as described in Section III. The cells have side $l$, and we assume that their bottom left corners have coordinates $(i \cdot l, j \cdot l)$ for $i, j \in \mathbb{Z}$. The cells are colored in a checkered fashion with $k^{2}$ colors, as shown in Figure 4. Each color can be identified by a pair $(a, b)$, with $a, b \in\{0,1, \ldots k-$ $1\}$; then the cell whose bottom left corner has coordinates $(i \cdot l, j \cdot l)$ has color $(i, j)(\bmod k)$. For each node $v, \operatorname{color}(v)$ is the color of the cell it belongs to. In the following we assume, w.l.o.g., that $k \geq 3$.

Given a value of $k$, we define communication graph $G_{k}$ as follows:

Definition $6\left(\right.$ graph $\left.G_{k}\right)$ : Communication graph $G_{k}$ includes all and only directed links $(u, v)$ such that $u$ is either the source or a leader node, and the SINR value at node $v$ relative to the signal transmitted by node $u$ is at least $\beta$, under the assumption that all leader (or source) nodes of color color $(u)$ are transmitting simultaneously.

In other words, graph $G_{k}$ contains all and only the links that can be used to broadcast packets when the broadcasting scheme in Figure 3 is used and the number of colors is $k^{2}$.

We now prove some fundamental properties of the broadcasting scheme in Figure 3:

Theorem 1: The broadcasting scheme defined in Figure 3 satisfies the following properties:

$i$ ) the source $s$ generates broadcast packets with rate $\frac{W}{k^{2}}$;

ii) let $p(i)$ be the packet generated at time $t_{i}$ by node $s$ during period $i$; if communication graph $G_{k}$ is cell connected w.r.t. $s$, packet $p(i)$ is received by all nodes in $\mathcal{V}-\{s\}$ within time $O\left(t_{i}+\frac{d(n)}{r_{\max }}\right)$.

Proof: We recall that we are assuming a time-slotted approach at the MAC layer, that time slots are colored using $\bar{k}^{2}$ colors, and that slot coloring is periodic with period $\bar{k}^{2}$.

Property $i$ ) is straightforward: the source node $s$ generates a new broadcast packet each time a slot with color color $(s)$ occurs, and thus gets $\frac{1}{k^{2}}$ of the available channel capacity $W$. To prove property $i i$ ), we first show that $a$ ) any leader node at cell distance $j \geq 0$ from the source node $s$ transmits packet $p(i)$ within period $i+j$. We proceed by induction on $j$. The base case $j=0$ (i.e., the transmitting node is the source) is

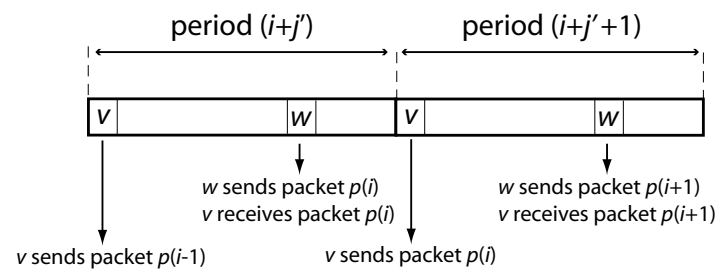

Fig. 5. Transmission opportunities in consecutive periods.

straightforward. Let us now consider the inductive case. Let $v$ be a leader node at cell distance $j+1$ from the source. By cell connectivity, there exists a path $P$ in the communication graph $G_{k}$ such that all nodes in $P-\{v\}$ have cell distance at most $j$. Observe that, by definition of $G_{k}$, path $P$ is composed only of leader nodes. Assume w.l.o.g. that path $P$ has minimal hop-length among the paths satisfying the condition for cell connectivity. Let $w$ denote the only node in $P$ such that link $(w, v)$ is in $G_{k}$. Since $c d(w, s) \leq j$, by induction hypothesis node $w$ transmits packet $p(i)$ within period $i+j^{\prime}$, with $j^{\prime} \leq j$. Since edge $(w, v)$ is in $G_{k}$, packet $p(i)$ is correctly received by node $v$ during period $i+j^{\prime}$. Hence, when node $v$ has its own transmission opportunity during period $i+j^{\prime}+1 \leq i+j+1$, packet $p(i)$ is in the transmission buffer and is transmitted (see Figure 5). Note that step 12. of the broadcasting scheme requires that node $v$ has transmitted packet $p(i-1)$ before being able to store packet $p(i)$ in the transmit buffer. This is actually the case, since, by induction hypothesis, packet $p(i-1)$ is transmitted by node $v$ during period $i+j^{\prime}$. We then conclude that node $v$ transmits packet $p(i)$ within period $i+j+1$, and property $a$ ) is proved. The following property immediately follows from property $a$ ) above and cell connectivity: $b$ ) packet $p(i)$ generated by source node $s$ during period $i$ is received by all nodes at cell distance $\leq j$ from $s$ by the end of period $i+j-1$, for any $j \geq 1$.

We now observe the following: 1 . since $k$ is a constant and broadcast packets can be sent in a constant amount of time, the duration of a period does not depend on the number $n$ of network nodes; 2 . the maximum (Euclidean) distance between $s$ and any other node in $\mathcal{V}-\{s\}$ is at most $d(n)$, and 3 . given that the square lattice step $l$ is within a constant factor from $r_{\max }$, the cell distance between two nodes at Euclidean distance $d$ is $O\left(\frac{d}{r_{\max }}\right)$. Given 2., 3., and property $b$ ), we have that a packet sent by the source during period $i$ is received by each node in $\mathcal{V}-\{s\}$ within $O\left(\frac{d(n)}{r_{\max }}\right)$ periods; this, combined with 1., implies property $i i$ ), and the theorem is proved.

We now prove the following fundamental lemma, which shows that there exists a constant value $\bar{k}$ of the number of colors such that any packet sent by a node in our broadcasting scheme is correctly received by all the nodes in the 8 surrounding cells. This implies that communication graph $G_{\bar{k}}$ satisfies cell adjacency, and cell connectivity of graph $G_{\bar{k}}$ is guaranteed in network deployments characterized in claims 2 and 3 and Corollary 1.

Lemma 2: Assume a cell partitioning as defined in Section III. There exists a constant value $\bar{k}$ of the number of colors such that, for each $k \geq \bar{k}$, every packet sent by a node in the broadcasting scheme is correctly received by all nodes in adjacent cells. 
The proof of the lemma is similar to the proofs of similar results proved, e.g., in [12], [13]. The interested reader can find the formal proof in the Appendix.

Note that, in accordance with the theoretical findings of [12], the value of $\bar{k}$ is independent of node density, but depends only on the step of the square lattice and on the path-loss exponent.

By observing that the capacity and latency bounds provided by our broadcasting scheme match the corresponding bounds stated in Section IV when $k=\bar{k}$, we have the following theorem, which is the main result of this section:

Theorem 2: The broadcasting scheme defined in Figure 3 provides asymptotically optimal broadcast capacity and latency for single broadcast source $s$ under the assumption that graph $G_{\bar{k}}$ is cell connected w.r.t. $s$.

\section{MATCHING CAPACITY AND LATENCY BOUNDS: THE MULTIPLE SOURCE CASE}

In this section we show how to extend the results of the previous section to the case of arbitrary set of broadcast sources. The broadcast sources are arbitrarily located within the network, and can arbitrarily share the aggregate network capacity, according to a bandwidth allocation vector $\mathcal{B}=$ $\left\{b_{1}, \ldots, b_{n}\right\}$. Assume the $b_{i}$ are defined as follows. For each node $i$, we assign an arbitrary, constant integer value $w_{i} \geq 0$ (which is defined as 0 if node $i$ is not an active broadcast source). The bandwidth share $b_{i}$ of node $i$ is defined $^{7}$ as

$$
b_{i}=\frac{w_{i}}{\sum_{j=1}^{n} w_{j}} .
$$

Define $\omega=\sum_{j=1}^{n} w_{j}$. We model broadcast source $s_{i}$, with $1 \leq i \leq n$ and $w_{i}>0$, as the composition of $w_{i}$ unitary broadcast sources (unitary source for short) $s_{i}^{1}, \ldots, s_{i}^{w_{i}}$. The collection of all the unitary broadcast sources is denoted $u s_{1}, \ldots, u s_{\omega}$, where in general $\omega$ might be larger than $n$. The location of a unitary source $u s_{j}$ is the physical location of the corresponding broadcast source $s_{i}$. With a slight abuse of notation, we use notation $\operatorname{cell}\left(u s_{j}\right)$ to denote the cell to which the broadcast source $s_{i}$ corresponding to the unitary source $u s_{j}$ belongs. Our broadcast scheme is designed to have each unitary source to generate a new broadcast packet in the network every $\omega$ communication rounds, thus satisfying proportionally fair allocation of the broadcast capacity.

Each communication round is composed of two phases: the convergecast and the broadcast phase. In turn, each phase is composed of $\bar{k}^{2}$ transmission slots, where the mechanism used to assign colors (transmission slots) to cell leaders is the same as the one used in the case of single broadcast source. The purpose of the first phase is to make all the new generated packets converge toward a specific, selected node $S$ (note that $S$ is not necessarily an active broadcast source). In the second phase, node $S$ disseminates all the packets received through convergecast using a single source broadcast, by means of the method we have described in the previous section. To achieve perfect separation between convergecast and broadcast

\footnotetext{
${ }^{7}$ In the definition, we assume that at least one broadcast source is active, i.e., at least one of the $w_{j}$ s is greater than 0 .
}

phases, each node is endowed with two separate, single-packet buffers: the convergecast buffer and the broadcast buffer.

The main idea is to use the convergecast phase to let node $S$ receive a new broadcast packet to transmit at each communication round (except for the initial rounds needed to fill the pipeline). This way, aggregate broadcast capacity is halved - new broadcast packets are injected only in one of the two equally long phases composing a communication round - with respect to the single source case, and remains asymptotically optimal. Furthermore, the convergecast scheme is designed in such a way that a packet generated by a source $u s_{i}$ at cell distance $d_{i}$ from $S$ during round $t$, is received by node $S$ exactly at round $t+d_{i}$, and broadcast in the network through node $S$ during round $t+d_{i}+1$. This implies that the latency of a broadcast packet is increased by an additive $O\left(\frac{d(n)}{r_{\max }}\right)$ term with respect to the latency in the case of single broadcast source, i.e., it remains asymptotically optimal.

We now show how to construct a convergecast scheme that satisfies both properties described above. For ease presentation, we cyclically number communication rounds from 0 to $(\omega-1)$, and assume all mathematics is modulo $\omega$. The convergecast and broadcast phase of a communication round are numbered similarly.

Given the positions of the $\omega$ unitary sources $u s_{i}$, we choose for each $u s_{i}$ the shortest path in the cell graph which leads to $\operatorname{cell}(S)$. In case more than one such paths exist, we arbitrarily pick one of them. The composition of these paths is a shortest path tree rooted at $\operatorname{cell}(S)$, connecting $\operatorname{cell}(S)$ to each of the cell $\left(u s_{i}\right)$ s through shortest paths. Starting from this tree, we build a simplified shortest path tree as follows. With a slight abuse of notation, in the following we denote by $\operatorname{cell}(T)$ the cell corresponding to tree node $T$. Each internal node with a single child in the tree is removed. Thus, the simplified shortest path tree contains only leaves, and internal nodes with at least two children. Edge $(U, V)$ in the simplified shortest path tree is labeled with $\operatorname{cd}(\operatorname{cell}(U), \operatorname{cell}(V))$, i.e., with the cell distance between the cells corresponding to the edge endpoints. An example of shortest path tree, and the corresponding simplified shortest path tree, is reported in Figure 6. Unless otherwise stated, in the following by shortest path tree we mean simplified shortest path tree.

Note that the leaves of the shortest path tree correspond to cells containing a unitary source, while internal nodes correspond to cells that do not necessarily contain a unitary source. If the cell corresponding to an internal node $T$ contains one or more unitary sources, we can model each of such sources as a leaf node with $T$ as parent node, and set the corresponding edge weight (cell distance) to 0 . Thus, in the following we assume without loss of generality that all unitary sources are represented as leaf nodes in the shortest path tree. Internal nodes in the shortest path tree act as forwarders towards the upper levels of the tree. When forwarding, one of the nodes within the corresponding cell (the cell leader) will be in charge of propagating broadcast packets up in the tree.

The designed convergecast scheme forces a synchronization of the unitary sources in order to avoid buffering at intermediate nodes (thus preserving asymptotically optimal broadcast 

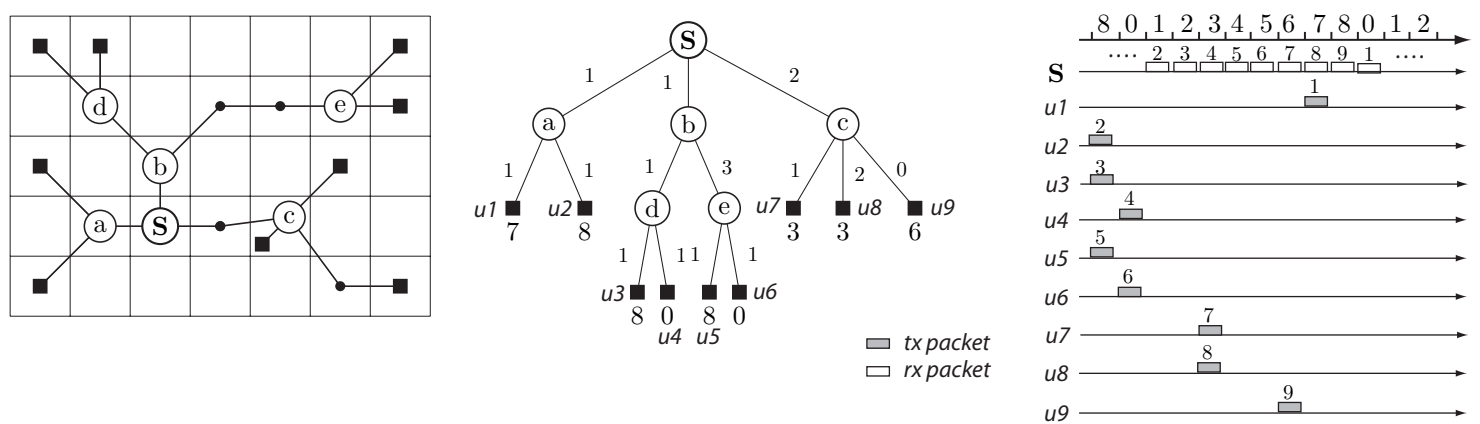

Fig. 6. Tree construction in the convergecast phase. Active unitary sources are denoted by a square. Cells at which two or more shortest paths intersect are denoted by a circled letter. Non-intersecting cells on the shortest paths are denoted by small circles. In the simplified shortest path tree (center), each unitary source is labeled with the round (modulo 9) at which a new packet is transmitted. The resulting transmission schedule is shown on the right.

latency), and can be recursively described as follows.

Let us consider an internal tree node $T$, and let us assume that $T$ has $z$ incoming edges, hence $z$ subtrees, containing $v$ unitary sources overall. Each subtree of $T$ can be either a single leaf or a proper tree. Let us assume that the parent of node $T$ in the tree requests $T$ to send a new broadcast packet from round $t$ to round $t+v-1$. Let the distances labeling the edges connecting $T$ to the subtrees be equal to $d_{1}, d_{2}, \ldots$, $d_{z}$, and let $m_{i}$ be the number of incoming new packets from edge $i$ in a complete set of $\omega$ convergecast rounds. Clearly $\sum_{i=1}^{z} m_{i}=v$.

In order to obtain perfect synchronization, and to be able to resend a new broadcast packet at each time step with constant size buffering, node $T$ requests each subtree to send packets in specific, non-overlapping communication rounds. In particular, the $i$-th subtree should send its messages in such a way they reach $T$ in $m_{i}$ consecutive rounds, from round

$$
\alpha_{i}=t+\sum_{j}^{i-1} m_{j} \quad \text { to } \quad \beta_{i}=t+\sum_{j}^{i} m_{j}-1
$$

where round numbering is performed cyclically, modulo $\omega$.

Given the delays $d_{i}$ determined by cell distances, and recursively assuming that the packet transmitted (either generated or forwarded) by a node at cell distance $d_{i}$ from $\operatorname{cell}(T)$ at round $t$ are received by nodes in $\operatorname{cell}(T)$ at round $t+d_{i}$, we have that the root of the $i$-th subtree must send its $m_{i}$ messages in the time steps $\alpha_{i}-d_{i}$ to $\beta_{i}-d_{i}$.

In practice, each subtree is requested to send its new packets in a specific time window, which is computed in such a way that the delays from $T$ to the roots of the subtrees make the messages reach $T$ in a continuous flow, from step $t$ to step $t+v-1$.

It is now clear that this mechanism can be repeated at each level of the tree, starting from the root $S$. The requirement of $S$ is to receive the $v=\omega$ messages from its subtrees at the contiguous steps 0 to $\omega-1$. This request is then recursively propagated toward the leafs. At the end of the process each leaf, i.e., each unitary source, knows at which communication round (of every cycle of $\omega$ communication rounds) it should send its new packet. See Figure 6 for an example of convergecast schedule computation.
We now prove that our proposed combined convergecast/broadcast scheme provides asymptotically optimal broadcast capacity and latency.

Lemma 3: Assume the above described convergecast scheme is used. The packet generated by a unitary source $u s_{i}$ at cell distance $d_{i}$ from node $S$ during the convergecast round $t$ is received by node $S$ during convergecast round $t+d_{i}$.

Proof: We prove the lemma by induction on the structure of the shortest path tree. More specifically, we prove that, given an arbitrary internal tree node $T$, the packet generated at round $t$ by a unitary source $u s_{i}$ in the subtree rooted at $T$ is received by node $T^{8}$ at round $t+d_{i}$, and re-transmitted up in the tree at round $t+d_{i}+1$.

The convergecast scheme is designed so that packets generated by unitary sources arrive at node $T$ in consecutive rounds. Denote by $v$ the total number of unitary sources in the subtree rooted at $T$, and let $t, \ldots, t+v$ be the round interval at which node $T$ wants to receive the packets generated by the unitary sources. The convergecast scheme dictates that the $i$-th child of $T$ sends its messages in such a way that they reach $T$ in $m_{i}$ consecutive rounds, where $m_{i}$ is the number of unitary sources in the subtree rooted at the $i$-th child of $T$. The endpoints of this interval are round $\alpha_{i}$ and $\beta_{i}$, as defined in (1). We now have two cases: $a$ ) the $i$-th child of $T$ is a leaf, and $b$ ) the $i$-th child of $T$ is an internal node. In case $a$ ), the $i$-th child of $T$ is a unitary source, denoted $u s_{i}$, which is connected to $T$ through a path of length $d_{i}$ in the cell graph $\left(d_{i}=0\right.$ in case the unitary source is located in $\operatorname{cell}(T))$. Since all cells in the path from $\operatorname{cell}\left(u s_{i}\right)$ to $\operatorname{cell}(T)$ do not contain unitary sources, and given that the leader node of any cell has a transmit opportunity in every convergecast round (recall the coloring scheme), it is sufficient to have $u s_{i}$ transmits its packet at time $t^{\prime}=\alpha_{i}-d_{i}$. This ensures that the packet generated by $u s_{i}$ at time $t^{\prime}$ is received by $T$ at time $t^{\prime}+d_{i}=$ $\alpha_{i}$. In case $\left.b\right)$, denote by $I$ the $i$-th child of node $T$, and let $d_{I}$ be the cell distance between $\operatorname{cell}(I)$ and $\operatorname{cell}(T)$. By induction hypothesis, the packet transmitted at time $t^{\prime \prime}$ by any unitary source $u s_{j}$ in the subtree rooted at $I$ is received by node $I$ at time $t^{\prime \prime}+d_{j I}$, where $d_{j I}$ is the cell distance between $\operatorname{cell}\left(u s_{j}\right)$ and $\operatorname{cell}(I)$. The convergecast scheme ensures that

\footnotetext{
${ }^{8}$ With a slight abuse of terminology, by "received by node $T$ " we mean "received by all nodes belonging to $\operatorname{cell}(T)$ ".
} 
all packets generated by unitary sources in the subtree rooted at $I$ arrive at $I$ in consecutive rounds, i.e., they do not conflict at $I$. Hence, the packet arrived at $I$ at a certain round $t$ can be transmitted at the next round $t+1$, i.e., the packet generated by $u s_{j}$ at round $t^{\prime \prime}$ is transmitted by $I$ at round $t^{\prime \prime}+d_{j I}+1$. Given that the cells in the path connecting $\operatorname{cell}(I)$ to $\operatorname{cell}(T)$ do not contain unitary sources, a packet transmitted by $I$ at time $t^{\prime \prime}+d_{j I}+1$ is received by $T$ at time $t^{\prime \prime}+d_{j I}+d_{I}=t^{\prime \prime}+d_{j}$, where $d_{j}$ is the cell distance between $u s_{j}$ and node $T$. Thus, we have formally proved that the property stated in the lemma holds for arbitrary nodes in the shortest path tree. The lemma then follows by considering the root node $S$ in the tree.

Lemma 4: Assume packets are broadcast using the combined convergecast/broadcast scheme described above, and assume graph $G_{\bar{k}}$ is cell connected w.r.t. node $S$. Then, the packet generated by a unitary source at time $t$ is received by all network nodes within time $t+O\left(\frac{d(n)}{r_{\max }}\right)$.

Proof: The packet generated by a unitary source is broadcast to all network nodes in two phases: 1) delivery to node $S$ (convergecast) and 2) broadcasting from node $S$. Given the properties of single source broadcasting, the fact that $G_{\bar{k}}$ is cell connected w.r.t. $S$, and considering that convergecast and broadcast phases have the same duration and are interleaved, we have that the packet latency during 2) is at most twice as much as the latency of single source broadcasting, i.e., it is $O\left(\frac{d(n)}{r_{\max }}\right)$. It remains to prove that also packet latency during phase 1) is $O\left(\frac{d(n)}{r_{\max }}\right)$. To this purpose, we first observe that cell connectivity w.r.t. node $S$ implies that, given any node $u$ in $\mathcal{V}-\{S\}$, there exists at least one path $P$ connecting $u$ and $S$ in $G_{\bar{k}}$ such that for all nodes $w$ in $P-\{S\}, c d(u, w)<c d(u, S)$. This property (call it property $a)$ ) is trivially implied by the fact that all links in $G_{\bar{k}}$ are bi-directional and the notion of cell distance is symmetric, hence existence of a forward "cell connected" path between $S$ and $u$ implies existence of a backward "cell connected" path between $u$ and $S$. By applying similar arguments to those in the proof of Theorem 1, property a) implies asymptotically optimal latency during phase 1 ), namely that the packet generated by a unitary source at time $t$ is received by node $S$ within time $t+O\left(\frac{d(n)}{r_{\max }}\right)$. This concludes the proof of the lemma.

Lemma 5: Assume packets are broadcast using the combined convergecast/broadcast scheme described above. The aggregate rate at which new broadcast packets are injected in the network is $\Theta(W)$.

Proof: The convergecast and broadcast phase have equal duration, hence the aggregate rate at which new broadcast packets are generated is half of the rate during the broadcast phase, which is $\Theta(W)$ (see Theorem 1), i.e., it remains $\Theta(W)$.

The theorem below, which is the main result of this paper, trivially follows by combining Lemma 3, Lemma 4, and Lemma 5.

Theorem 3: The combined convergecast/broadcast scheme described above provides asymptotically optimal broadcast capacity and latency, under the assumption that graph $G_{\bar{k}}$ is cell connected w.r.t. an arbitrary node $S$.

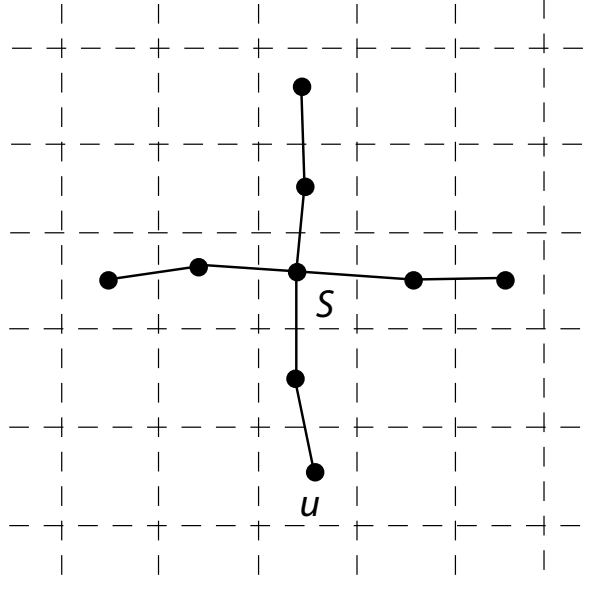

Fig. 7. Network topology which satisfies cell connectivity w.r.t. node $S$, but not w.r.t. node $u$.

It is worth observing that the above theorem holds under the assumption that $G_{\bar{k}}$ is cell connected w.r.t. an arbitrary node $S$. Hence, the network topology requirement is much looser than in the case of the broadcast scheme of Figure 3 , which required cell connectivity with respect to a specific node (the broadcast source). For instance, consider the network reported in Figure 7, and assume node $u$ is the only broadcast source. In this case, the broadcast scheme of Figure 3 cannot guarantee asymptotically optimal latency, since the network is not cell connected w.r.t. $u$. However, if we use the combined convergecast/broadcast scheme presented in this section, packets generated by node $u$ can be convergecast to node $S$, and then broadcast in the network starting from $S$. Since the network is cell connected w.r.t. $S$, Theorem 3 implies that asymptotically optimal broadcast capacity and latency can be achieved. The price to pay to have optimal guarantees on packet delivery latency is a reduced broadcast rate, which is halved with respect to that achieved by the broadcast scheme of Figure 3.

\section{DISCUSSION AND PRACTICAL CONSIDERATIONS}

The results presented in this paper have shown that, contrary to what happens in case of unicast transmissions, broadcasting in wireless multi-hop networks is not relay limited. This discrepancy originates from the fact that a single wireless communication is potentially correctly received by all nodes within transmission range. While in case of unicast only one of these potentially many receivers is actually interested in the packet, and all the other nodes treat the incoming signal as interference, in case of broadcast all potential receivers are interested in receiving the packets.

It is interesting to compare the throughput/delay values achieved by our optimal broadcasting scheme with those dictated by the optimal throughput/delay tradeoff in case of unicast communication derived in [4], [5]. First, we notice that the notion of throughput in broadcast and unicast transmission is slightly different, since in the former case one is interested in characterizing the aggregate rate of all broadcast sources, while in unicast transmission the quantity of interest is the per-node throughput. In case of random networks 
(the network setting considered in [4], [5]), our broadcasting scheme achieves a throughput $T(n)=\Theta(1)$ and a delay of $D(n)=\Theta\left(\frac{d(n)}{r_{\max }}\right)=\Theta\left(\sqrt{\frac{n}{\log n}}\right)$. In case of unicast communication, a throughput of $T(n)=\Theta(1)$ implies a delay value of $D(n)=\Theta(n)$, which is much larger than the value obtained with broadcast communication. Thus, our results seem to indicate that a much more favorable throughput/delay tradeoff can be obtained with broadcast than in the case of unicast transmission.

We also want to mention that, up to tedious technical details, the results presented in this paper can be extended to a network setting in which nodes use different transmission power, as long as the ratio between the largest and smallest transmission power used by nodes is an arbitrary constant. Furthermore, our results can be proved to hold also under the cost-based radio propagation model of [22], which is shown to closely resemble the log-normal shadowing model.

While the results presented in this paper are mostly interesting from a theoretical point of view, they also have implications on the design of practical broadcast schemes. In particular, our results suggest that, if some topological properties of the communication graph are satisfied (cell connectivity), latency and capacity optimal broadcasting can be achieved by:

a) properly partitioning the network deployment region;

b) selecting a leader node in each cell of the partitioning;

c) assigning a color to each cell;

d) executing a simple (convergecast/)broadcasting scheme.

The partitioning is used to spatially separate concurrent transmissions, so that they do not corrupt each other. While in this paper we have used a square partitioning, the actual partitioning used in a practical scenario mainly depends on the features of the radio propagation environment. On the other hand, the shape of the partitioning does not depend on node density, as long as the cell connectivity property is guaranteed.

Steps $a$ ) and $b$ ) can be easily accomplished if nodes are aware of their location, e.g., if they are equipped with GPS receivers. GPS receivers are becoming increasingly present in smart cellular phones and PDAs, and are standard equipment in some types of wireless networks (e.g., vehicular networks) or wireless sensor networks. While the techniques presented in this paper are based on absolute node locations, they in principle can be extended to deal with virtual coordinate systems, such as that proposed in [19] for wireless sensor networks.

For what concerns $b$ ), the results presented in this paper are based on the implicit assumption that the overhead for electing leader nodes consumes a negligible share of the available network capacity and does not impact broadcast latency. This assumption is straightforwardly satisfied if leader nodes are, e.g., selected once and for all at the beginning of the network operational lifetime. However, in many cases the role of leader node should be rotated based on time evolving network properties, e.g., node battery levels, node mobility, etc. In these situations, the message overhead induced by the leader election process should be carefully estimated. For instance, the SCREAM primitive proposed in [2] can be used to complete the leader election process in $O(\log n)$ steps, which implies that asymptotically optimal broadcast capacity and latency is still possible in a homogeneous network as long as the leader election process is repeated at most once every $O(\log n)$ repetitions of the broadcast scheme.

Concerning $c$ ), we observe that coloring is based on the assumption that a spatial TDMA scheme is used at the MAC layer. Hence, a relatively tight time synchronization mechanism should be implemented. Time synchronization is typically available in some application scenarios, such as wireless sensor networks. More in general, we observe that time synchronization is relatively easy to achieve if nodes are equipped with GPS receivers. Once time synchronization is achieved, building the communication schedule is straightforward, and can be easily implemented in a fully distributed environment in the case of single broadcast source: all a node needs to know is whether it is acting as the leader node in its cell, and what is the cell color. The case of arbitrary broadcast source set is more complex, as it requires network-wide coordination of broadcast sources for sending the respective packets. Similarly to the case of leader election, if the broadcast source set (and relative proportional capacity allocation) does not change with time, coordination of the broadcast sources can be determined once and for all at the beginning of the network operation, and broadcast optimality is not compromised. In presence of dynamic broadcast source set and/or capacity allocation, coordination of the broadcast sources should be periodically re-determined, and the induced message overhead should be carefully estimated.

\section{CONCLUSIONS}

In this paper, we have investigated the fundamental question of whether asymptotically optimal broadcast capacity and latency can be simultaneously achieved in a wireless multihop network. To answer this question, we have introduced a novel topological notion of graph connectivity, called cell connectivity, and shown that asymptotically optimal capacity and latency can be achieved in a cell connected network, even in presence of an arbitrary number of broadcast sources that arbitrarily share the broadcast capacity.

Several issues are left open by this paper, such as investigating whether our results can be extended to mobile networks. Initial results in this direction are presented in [21]. Furthermore, whether the broadcasting scheme presented in this paper can be generalized to achieve a (possibly optimal) throughput/delay tradeoff is another question of interest. Finally, understanding whether cell connectivity, which is a sufficient condition for having asymptotically optimal broadcast latency, is also a necessary condition is another question left open by this paper.

\section{REFERENCES}

[1] N. Bansal, Z. Liu, "Capacity, Delay and Mobility in Wireless Ad-Hoc Networks", Proc. IEEE Infocom, pp. 1553-1563, 2003.

[2] G. Brar, D. Blough, P. Santi, "The SCREAM Approach for Efficient Distributed Scheduling with Physical Interference in Wireless Mesh Networks, Proc. IEEE International Conference on Distributed Computing Systems (ICDCS), pp. 214-224, 2008. 
[3] O. Dousse, P. Thiran, "Connectivity vs Capacity in Dense Ad Hoc Networks", Proc. IEEE Infocom, pp. 476-486, 2004.

[4] A. El Gamal, J. Mammen, B. Prabhakar, D. Shah, "Optimal Throughput-Delay Scaling in Wireless Networks-Part I: The Fluid Model", IEEE Trans. on Information Theory, Vol. 52, n. 6, pp. 2568-2592, 2006.

[5] A. El Gamal, J. Mammen, B. Prabhakar, D. Shah, "Optimal Throughput-Delay Scaling in Wireless Networks-Part II: Constant-Size Packets", IEEE Trans. on Information Theory, Vol. 52, n. 11, pp. 5111-5116, 2006.

[6] R.B. Ellis, J.L. Martin, C. Yan, "Random Geometric Graph Diameter in the Unit Ball", Algorithmica, Vol. 47, n. 4, pp. 421-438, 2007.

[7] M. Franceschetti, M.D. Migliore, P. Minero, "The Capacity of Wireless Networks: Information-theoretic and Physical Limits", Internet draft, extended version of a paper appeared on Proc. Allerton Conf. 2007, available at http: / / circuit. ucsd. edu/ massimo/papers.html.

[8] R. Gandhi, S. Parthasarathy, A. Mishra, "Minimizing Broadcast Latency and Redundancy in Ad Hoc Networks", Proc. ACM MobiHoc, pp. 222-232, 2003.

[9] M. Grossglauser, D.N.C. Tse, "Mobility Increases the Capacity of Ad-Hoc Wireless Networks", Proc. IEEE Infocom, pp. 13601369, 2001.

[10] P. Gupta and P.R. Kumar, "The Capacity of Wireless Networks," IEEE Transactions on Information Theory, Vol. 46, No. 2, pp. 388-404, 2000.

[11] S.C.H. Huang, P.J. Wan, J. Deng, Y.S. Han, "Broadcast Scheduling in Interference Environment", IEEE Transactions on Mobile Computing, Vol. 7, n. 11, pp. 1338-1348, 2008.

[12] A. Keshavarz-Haddad, R. Riedi, "On the Broadcast Capacity of Multihop Wireless Networks: Interplay of Power, Density and Interference”, Proc. IEEE SECON, pp. 314-323, 2007.

[13] A. Keshavarz-Haddad, V. Ribeiro, R. Riedi, "Broadcast Capacity in Multihop Wireless Networks", Proc. ACM Mobicom, pp. 239-250, 2006.

[14] V.F. Kolchin, B.A. Sevast'yanov, and V.P. Chistyakov, Random Allocations, V.H. Winston and Sons, Washington D.C., 1978.

[15] M.J. Neely, E. Modiano, "Capacity and Delay Tradeoffs for Ad Hoc Mobile Networks", IEEE Trans. on Information Theory, vol. 51, n. 6, pp. 1917-1937, 2005.

[16] A. Ozgur, O. Leveque, D. Tse, "Hierarchical Cooperation Achieves Linear Capacity Scaling in Ad Hoc Networks", Proc. IEEE Infocom, pp. 382-390, 2007.

[17] A. Ozgur, O. Leveque, "Throughput-Delay Trade-off for Hierarchical Cooperation in Ad Hoc Wireless Networks", IEEE Trans. on Information Theory, Vol. 56, n. 3, pp. 1369-1377, 2010.

[18] M.K. Park, V. Rodoplu, "Energy Maps for Mobile Wireless Networks: Coherence Time versus Spreading Period", IEEE Transactions on Mobile Computing, to appear, 2009.

[19] A. Rao, C.H. Papadimitriou, S. Shenker, I. Stoica, "Geographic Routing without Location Information", Proc. ACM Mobicom, pp. 96-108, 2003.

[20] T.S. Rappaport, Wireless Communications, Prentice Hall, Upper Saddle River, NJ, 2002.

[21] G. Resta, P. Santi, "On the Fundamental Limits of Broadcasting in Wireless Mobile Networks", Proc. IEEE Infocom (miniconference), 2010.

[22] C. Scheideler, A. Richa, P. Santi, “An O(log n) Dominating Set Protocol for Wireless Ad Hoc Networks under the Physical Interference Model, Proc. ACM MobiHoc, pp. 91-100, 2008.

[23] G. Sharma, R. Mazumdar, N. Shroff, "Delay and Capacity Trade-offs in Mobile Ad Hoc Networks: A Global Perspective", Proc. IEEE Infocom, pp. 1-12, 2006.

[24] S. Toumpis, A. Goldsmith, "Large Wireless Networks under Fading, Mobility and Delay Constraints", Proc. IEEE Infocom, pp. 610-619, 2004.

[25] R. Zheng, "Information Dissemination in Power-Constrained Wireless Networks", Proc. IEEE Infocom, pp. 1-10, 2006.

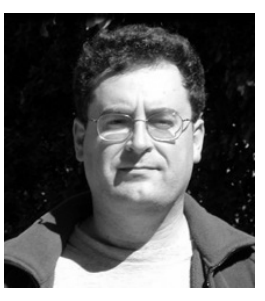

G. Resta received the MS degree in computer science from the University of Pisa, Italy, in 1988. In 1996, he became a researcher at the Istituto di Matematica Computazionale of the Italian National Research Council (CNR), Pisa. He is now a senior researcher at the Istituto di Informatica e Telematica (CNR) in Pisa. His research interests include computational complexity (especially in relation to linear algebra problems), parallel and distributed computing, and the study of structural properties of wireless ad hoc networks.

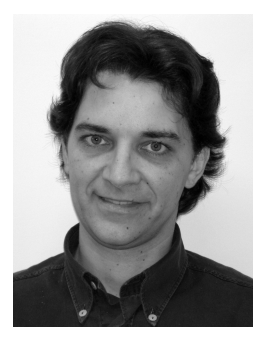

P. Santi received the Laura Degree and Ph.D. degree in computer science from the University of Pisa in 1994 and 2000, respectively. He has been with the Istituto di Informatica e Telematica del CNR in Pisa, Italy, since 2001, first as reasearcher and now as senior researcher. During his career, he visited Georgia Institute of Technology in 2001, and Carnegie Mellon University in 2003. His research interests include fault-tolerant computing in multiprocessor systems (during $\mathrm{PhD}$ studies), and, more recently, the investigation of fundamental properties of wireless multihop networks such as connectivity, lifetime, capacity, mobility modeling, and cooperation issues. He has contributed more than 60 papers and a book in the field of wireless ad hoc and sensor networking, he is Associate Editor of IEEE Trans. on Mobile Computing and IEEE Trans. on Parallel and Distributed Systems, he has been General Co-Chair of ACM VANET 2007 and 2008, and he is involved in the organizational and technical program committee of several conferences in the field. He is a member of IEEE Computer Society and a senior member of ACM and SIGMOBILE. 


\section{APPENDIX}

Proof of Lemma 1. The proof is based on the following result from [14] (Thm. 5, page 111):

Proposition 1: Assume $n$ nodes are distributed uniformly and independently at random into $C$ cells, and let $\eta=\frac{n}{C}$. If $\frac{\eta}{\log C} \rightarrow 1$ as $n, C \rightarrow \infty$ and $k=k(\eta, C)$ is chosen so that $k<\eta$ and $C p_{k} \rightarrow \lambda$, where $\lambda$ is a positive constant and $p_{k}=\frac{\eta^{k}}{k !} e^{-\eta}$, then $\operatorname{Prob}\{\operatorname{Min} \geq k\} \rightarrow 1$, where Min is the random variable denoting the number of nodes in the minimally occupied cell.

We first prove the lemma under the assumption that $j^{2}=$ $8 h^{2}$. It is easy to verify that for $j^{2}=8 h^{2}$ we are under the hypothesis of Proposition 1. In fact:

$$
\frac{\eta}{\log C}=\frac{\log n}{\log n-\log \log n} \rightarrow 1
$$

as $n \rightarrow \infty$. We now verify that $C p_{k} \rightarrow \lambda=1$ when $k=$ $k(n)=1$ (note that $k<\eta$, for any large enough value of $n$ ).

We have:

$$
C p_{k}=\frac{n}{\log n} \cdot \frac{\log n}{1 !} \cdot \frac{1}{e^{\log n}} \rightarrow 1
$$

when $n \rightarrow \infty$.

Hence, we can apply Proposition 1, which implies that each cell contains at least 1 node w.h.p.

The proof for the case $j^{2}>8 h^{2}$ is straightforward: by setting $j^{2}>8 h^{2}$, we distribute the same number $n$ of nodes into strictly fewer cells than in the case $j^{2}=8 h^{2}$. Hence, the minimum cell occupancy in this case cannot be lower than that in the case when $j^{2}=8 h^{2}$, which is at least 1 . This concludes the proof of the lemma.

Proof of Lemma 2. Let us fix

$$
k \geq \bar{k}=\left\lceil 2+2^{\frac{3}{2}+\frac{4}{\alpha}}\left(\beta \zeta(\alpha-1) h^{\alpha} /\left(h^{\alpha}-1\right)\right)^{\frac{1}{\alpha}}\right\rceil,
$$

where $\zeta$ is the Riemann's zeta function. We first observe that the broadcasting scheme ensures that in each cell there is at most one transmitting node.

We consider an arbitrary sender-receiver pair $(S, R)$, as in Fig. 4. We obtain the lower bound on $k$ by considering the minimum possible received power at $R$ of the signal transmitted by $S$, and deriving an upper bound to the total interference experienced at node $R$. The worst case, with respect to the power received by $R$ from $S$, is obtained when $R$ and $S$ are as far apart as possible, yet in adjacent cells. This happens when the two cells are diagonally adjacent, and the two nodes are in the two opposite corners of their respective cells. It follows that $d(S, R) \leq 2 \sqrt{2} l$, where $l$ is the side of a cell (note that condition $h>1$ in the definition of $l$ ensures that $\left.d(S, R)<r_{\max }\right)$. The received power $P_{R}(S)$ is thus at least $P /(2 \sqrt{2} l)^{\alpha}$.

Let us consider now the interference experienced by $R$ under the condition, ensured by our broadcasting scheme, that in each cell with the same color as $S$ there is at most one (in the worst case, exactly one) active transmitter. Assume w.l.o.g. that $\operatorname{cell}(S)$ has coordinates $(0,0)$. Given the coloring scheme, interferers lie in the cells with bottom left corner at $(x \cdot k$. $l, y \cdot k \cdot l)$ with $x, y \in \mathbb{Z}$ and $(x, y) \neq(0,0)$ (shaded cells in Figure 4).

The distance $d(x, y)$ between $R$ and an interferer located in cell $(x \cdot k \cdot l, y \cdot k \cdot l)$, with $x, y \neq 0$, can be lower bounded as follows:

$$
d(x, y) \geq \sqrt{\left(|x| k l-\mu_{1} l\right)^{2}+\left(|y| k l-\mu_{2} l\right)^{2}},
$$

where $\mu_{1}, \mu_{2} \in\{0,1,2\}$ depending on the actual cell (among the 8 surrounding $\operatorname{cell}(S)$ ) in which the receiver $R$ lies. We recall that we are lower bounding $P_{R}(S)$ and upper bounding interference, hence we can assume that $R$ is located in a diagonally adjacent cell when lower bounding $P_{R}(S)$, and that it is located in another, arbitrary, cell adjacent to $S$ when upper bounding interference.

Similarly, when either $x$ or $y$ are 0 , we have

$$
d(x, y) \geq \max \{|x| k l,|y| k l\}-\mu_{3} l
$$

where $\mu_{3} \in\{0,1,2\}$. Since $a^{2}+b^{2} \geq(\max \{a, b\})^{2}$, from (2) we obtain

$$
\begin{aligned}
d(x, y) & \geq \max \left\{|x| k l-\mu_{1} l,|y| k l-\mu_{2} l\right\} \\
& \geq \max \{|x| k l-2 l,|y| k l-2 l\}
\end{aligned}
$$

for $x, y \neq 0$. When either $x$ or $y$ is 0 , equation (3) implies

$$
d(x, y) \geq \max \{|x| k l,|y| k l\}-2 l \text {. }
$$

Hence, combining the two inequalities we obtain the following lower bound on $d(x, y)$, which is valid for each $(x, y) \neq(0,0)$ :

$$
\begin{aligned}
d(x, y) & \geq \max \{|x|,|y|\} k l-2 l=l(k \max \{|x|,|y|\}-2) \geq \\
& \geq(k-2) l \max \{|x|,|y|\} .
\end{aligned}
$$

Note that the last bound is always strictly positive, since we are assuming $k \geq 3$ and $|x|,|y|$ are not both 0 .

The interference received by $R$ thus satisfies

$$
\begin{aligned}
P_{I} & <\sum \frac{P}{((k-2) l \max \{|x|,|y|\})^{\alpha}}= \\
& =\frac{P}{(k-2)^{\alpha} l^{\alpha}} \sum \frac{1}{\max \{|x|,|y|\}^{\alpha}},
\end{aligned}
$$

where the sum is extended over all the pairs $(x, y) \neq(0,0)$, with $x, y \in \mathbb{Z}$.

Counting twice the contributions along $x=0, y=0$, and $|x|=|y|$, we have

$$
\sum_{(x, y) \neq(0,0)} \frac{1}{\max \{|x|,|y|\}^{\alpha}}<8 \sum_{x=1}^{\infty} \sum_{y=0}^{x} \frac{1}{x^{\alpha}}
$$

due to the 8-fold symmetry of the summation shown in Figure 8. Collecting the values for which $\max (x, y)=x$ we obtain

$8 \sum_{x=1}^{\infty} \sum_{y=0}^{x} \frac{1}{x^{\alpha}}=8 \sum_{x=1}^{\infty} \frac{x+1}{x^{\alpha}}<16 \sum_{x=1}^{\infty} \frac{1}{x^{\alpha-1}}=16 \zeta(\alpha-1)$,

where $\zeta(\cdot)$ is the Riemann's zeta function.

Summarizing we have

$$
P_{I}<\frac{16 P \zeta(\alpha-1)}{(k-2)^{\alpha} l^{\alpha}}
$$




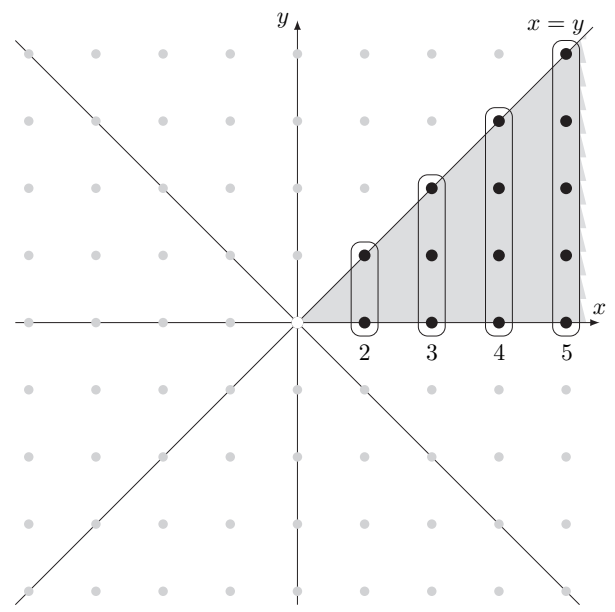

Fig. 8. 8-fold symmetry in the derivation of the upper bound to the total interference.

We now plug the value of $l=\frac{r_{\max }}{2 h \sqrt{2}}$ in the inequality

$$
\frac{P_{R}(S)}{N+P_{I}} \geq \beta
$$

and solve the inequality for $k$, obtaining

$$
k \geq \bar{k}=\left\lceil 2+2^{\frac{3}{2}+\frac{4}{\alpha}}\left(\beta \zeta(\alpha-1) \frac{h^{\alpha}}{h^{\alpha}-1}\right)^{\frac{1}{\alpha}}\right\rceil .
$$

Hence, for any value of $k \geq \bar{k}$, the message sent by node $S$ is correctly received at $R$, no matter how many simultaneous transmissions whose transmitters have the same color as $S$ are occurring. Given the arbitrary choice of the sender-receiver pair $(S, R)$, the lemma is thus proved. 